\title{
Fe Impurities Weaken the Ferromagnetic Behavior in Au Nanoparticles
}

\author{
P. Crespo, ${ }^{1,2}$ M. A. García, ${ }^{1,2}$ E. Fernández Pinel, ${ }^{1}$ M. Multigner,${ }^{1}$ D. Alcántara, ${ }^{3}$ J. M. de la Fuente, ${ }^{3}$ \\ S. Penadés, ${ }^{3}$ and A. Hernando ${ }^{1,2}$ \\ ${ }^{1}$ Instituto de Magnetismo Aplicado (RENFE-UCM-CSIC), P.O. Box 155, 28230 Las Rozas, Madrid, Spain \\ ${ }^{2}$ Dpto. Física de Materiales, UCM, 28040 Madrid, Spain \\ ${ }^{3}$ Grupo Carbohidratos, Lab. of Glyconanotechnology IIQ-CSIC, Américo Vespucio s/n, 41092 Sevilla, Spain
}

(Received 23 February 2006; published 26 October 2006)

In this Letter, we report on a crucial experiment showing that magnetic impurities reduce the ferromagnetic order temperature in thiol-capped $\mathrm{Au}$ glyconanoparticles (GNPs). The spontaneous magnetization of AuFe GNPs exhibits a fast decrease with temperature that contrasts with the almost constant value of the magnetization observed in Au NPs. Moreover, hysteresis disappears below $300 \mathrm{~K}$. Both features indicate that $\mathrm{Fe}$ impurities reduce the high local anisotropy field responsible for the ferromagnetic behavior in Au GNPs. As a consequence, the amazing ferromagnetism in Au NPs should not be associated with the presence of magnetic impurities.

The experimental observation of a ferromagnetic (FM) behavior, characterized by magnetic hysteresis up to room temperature (RT) in thiol-capped gold nanoparticles (NPs) [1] and giant magnetization values and anisotropy in thiolcapped Au thin films [2], is attracting a broad interest. First, there is a great challenge in understanding the physical mechanism inducing FM-like behavior in systems that are far from being ferromagnetic. Second, the possibility of combining nanoscale with permanent magnetism up to human body temperature and a good biocompatibility, as occurs for gold, opens a wide range of future biomagnetic applications such as ferrofluids in molecular imaging, tumor targeting, and hyperthermia.

The electronic properties of NPs are different from that of their bulk counterparts due to the so-called size and surface effects [3]. Hence, modifications of the magnetic behavior could be expected by decreasing particle size to the nanoscale and/or surface modification. One strategy is the modification and tuning of the electronic properties of NPs by appropriate functionalization [4]. Those changes have been experimentally observed by x-ray photoemission spectroscopy in alkanethiol stabilized Au NPs [5] and through the FM behavior of thiol-capped Au and Pd NPs $[1,6]$, both non FM in bulk state. The case of Au NPs is particularly interesting since bulk $\mathrm{Au}$ is diamagnetic. Moreover, this striking FM behavior is not observed when the Au NPs are stabilized with a surfactant. A similar tendency is observed in appropriate functionalized $\mathrm{Au}$ thin films [2].

From the above reported results, it turns out that the appearance of a magnetic moment should be associated to surface functionalization with strong interacting thiol derivatized species as well as with the reduction of particle size to the nanoscale. As inferred from extended x-ray absorption near-edge structure (XANES), capping promotes a charge transfer from $\mathrm{Au}$ atoms to organic chains $[1,4,7]$. Huge values of spin-orbit splitting, around $0.4 \mathrm{eV}$, have been measured in Au surfaces [8]. The localized character of the magnetic moments, inferred from surface plasmon resonance studies (SPR) [9], as well as its orbital character, which implies a high magnetic anisotropy, have been proposed as the mechanism responsible for the ferromagnetic-like behavior [10].

However, the possible influence of magnetic impurities is always an important concern. In this Letter, we report on the magnetic behavior of thiol-capped Au glyconanoparticles (GNPs) where Fe impurities have been introduced in a controlled way [11]. The properties will be compared with that exhibited by high purity Au NPs. From these results, it can be concluded that the FM behavior should be inherently associated with the modification on the electronic structure of Au NPs and not to the presence of FM impurities.

Thiol-capped AuFe and Au GNPs stabilized by maltose and lactose neoglycoconjugates with different $\mathrm{Au}: \mathrm{Fe}$, and $\mathrm{Au}: \mathrm{S}$ ratios have been studied. GNPs were prepared in aqueous solution by one step synthesis developed by Penadés et al. [11,12]. Table I summarizes the studied samples. Particle size distribution histograms were measured from transmission electron microscopy micrographs using an automatic image analyzer. Magnetic characterization has been carried out with a SQUID magnetometer. The magnetization of the NPs was obtained after subtracting the diamagnetic contribution of the sample holder. SPR has been measured by means of UV-VIS optical absorption.

TEM micrographs show monodispersed particles with an average particle size of around 1.8 and $2 \mathrm{~nm}$, respectively, for samples stabilized with maltose and lactose neoglycoconjugates [7,11]. From these values, the number of atoms in the cluster and in the surface is estimated following [13]. The average composition, obtained by inductively coupled plasma (ICP) analysis, and the percentage of $\mathrm{Au}$ atoms bonded to a thiol group (by assuming that each available $\mathrm{S}$ is bonded to a surface $\mathrm{Au}$ atom), is also 
TABLE I. Summary of the structural characteristics of Au and AuFe GNPs. The estimated values of the anisotropy constant are also included.

\begin{tabular}{|c|c|c|c|c|c|c|c|c|}
\hline Sample & Composition $^{\mathrm{a}}$ & $\begin{array}{c}\text { Av NP } \\
\text { size }(\mathrm{nm})^{\mathrm{b}}\end{array}$ & $\begin{array}{l}\text { Total Nr } \\
\% \mathrm{Nr}^{\mathrm{c}}\end{array}$ & $\begin{array}{c}\text { Av. } \\
\text { composition } \\
\text { of cluster } \\
\text { (at. } \%)\end{array}$ & Au:S & $\begin{array}{c}\% \text { surface } \\
\text { Au atoms } \\
\text { bonded }\end{array}$ & $\begin{array}{l}\text { Volume Au } \\
\text { bonded } \\
\left(\mathrm{nm}^{3}\right)(4)^{\mathrm{d}}\end{array}$ & $K\left(J / m^{3}\right)$ \\
\hline Au-SRmaltose & $\begin{array}{l}\left(\mathrm{C}_{23} \mathrm{H}_{44} \mathrm{O}_{11} \mathrm{~S}\right)_{201} \mathrm{Au}_{201} \\
27 \text { wt. \% Au }\end{array}$ & $1.8 \mathrm{~nm}$ & $\begin{array}{c}201 \text { at/cluster } \\
63.7 \%\end{array}$ & $\mathrm{Au}_{100}$ & $1: 1$ & $100 \%$ & 2.5 & $4 \times 10^{7}$ \\
\hline AuFe1-SRmaltose & $\begin{array}{l}\left(\mathrm{C}_{23} \mathrm{H}_{44} \mathrm{O}_{11} \mathrm{~S}\right)_{191} \mathrm{Au}_{191} \mathrm{Fe}_{10} \\
23.7 \text { wt. } \% \mathrm{Au} 0.3 \text { wt. } \% \mathrm{Fe}\end{array}$ & $1.8 \mathrm{~nm}$ & $\begin{array}{c}201 \text { at/cluster } \\
63.7 \%\end{array}$ & $\mathrm{Au}_{95} \mathrm{Fe}_{5}$ & $1: 1$ & $100 \%$ & 2.5 & $2 \times 10^{7}$ \\
\hline AuFe2-SRmaltose & $\begin{array}{l}\left(\mathrm{C}_{23} \mathrm{H}_{44} \mathrm{O}_{11} \mathrm{~S}\right)_{533} \mathrm{Au}_{198} \mathrm{Fe}_{3} \\
12.1 \text { wt.\% } \mathrm{Au} 0.06 \text { wt. } \% \mathrm{Fe}\end{array}$ & $1.8 \mathrm{~nm}$ & $\begin{array}{c}201 \text { at/cluster } \\
63.7 \%\end{array}$ & $\mathrm{Au}_{98.5} \mathrm{Fe}_{1.5}$ & $1: 2.7$ & $100 \%$ & 2.5 & $0.7 \times 10^{7}$ \\
\hline Au-SRlactose & $\begin{array}{l}\left(\mathrm{C}_{20} \mathrm{H}_{37} \mathrm{O}_{14} \mathrm{~S}\right)_{98} \mathrm{Au}_{314} \\
54 \text { wt.\% } \% \text { u }\end{array}$ & $2 \mathrm{~nm}$ & $\begin{array}{c}314 \text { at/cluster } \\
55.4 \%\end{array}$ & $\mathrm{Au}_{100}$ & $1: 0.3$ & $56.3 \%$ & 1.9 & $6 \times 10^{7}$ \\
\hline AuFe-SRlactose & $\begin{array}{l}\left(\mathrm{C}_{20} \mathrm{H}_{37} \mathrm{O}_{14} \mathrm{~S}\right)_{95} \mathrm{Au}_{266} \mathrm{Fe}_{48} \\
\text { 51 wt.\% Au } 2.8 \text { wt.\% Fe }\end{array}$ & $2 \mathrm{~nm}$ & $\begin{array}{c}314 \text { at/cluster } \\
55.4 \%\end{array}$ & $\mathrm{Au}_{84.7} \mathrm{Fe}_{15.3}$ & $1: 0.4$ & $54.6 \%$ & 1.9 & $2 \times 10^{7}$ \\
\hline
\end{tabular}

${ }^{\mathrm{a} C}$ Composition determined from ICP analysis.

${ }^{\mathrm{b}}$ Average particle size as calculated from particle size distribution histograms.

Total Nr: calculated number of atoms for a pure gold cluster. \% Nr: percentage of atoms located at the surface of a pure gold cluster.

${ }^{\mathrm{d}}$ Estimated volume corresponding to surface $\mathrm{Au}$ atoms bonded to $\mathrm{S}$.

indicated in Table I. Au $-\mathrm{L}_{3}$ edge XANES spectra indicates a charge transfer from Au atoms to the thiol derivatized molecule [7].

Magnetic hysteresis up to RT is observed for both $\mathrm{Au}$ GNPs, see Fig. 1, confirming previous results [1]. The amount of Fe impurities (below $00.01 \mathrm{ppm}$ ) is not enough to account for the magnetization values. Thus, the magnetization should arise from the charge transfer that takes place upon bonding. The inset shows a non negligible coercivity present even at RT, decreasing from around $100 \mathrm{Oe}$ at $5 \mathrm{~K}$ down to $30 \mathrm{Oe}$ at $300 \mathrm{~K}$. In the case of Au-SRLactose, a diamagnetic component is observed at RT (subtracted from the data plotted in Fig. 1), which is absent for Au-SRMaltose sample. According to the Au:S ratio, see Table I, not all surface Au atoms are bonded to a thiol group for the Au-SRLactose sample. Thus, a diamagnetic contribution arising from the core of the NP as well as from surface atoms not linked to thiol, and presumably from lactose, is resolved. When the magnetization is normalized to the amount of (estimated) $\mathrm{Au}$ bonded, see Table I, the magnetization values, at $5 \mathrm{~K}$, become much more similar, $M_{\mathrm{Au}-\mathrm{SRmaltose}}=0.134 \mathrm{emu} / \mathrm{g}$ and $M_{\text {Au-SRlactose }}=0.08 \mathrm{emu} / \mathrm{g}$. From these values, the magnetic moment of a bonded $\mathrm{Au}$ atom is estimated to be $\mu_{\mathrm{Au}}=0.0047 \mu_{B}$ and $\mu_{\mathrm{Au}}=0.003 \mu_{B}$, for $\mathrm{Au}-$ SRmaltose and Au-SRLactose, respectively. The quite similar values of the $\mu_{\mathrm{Au}}$ provide further evidence that the appearance of a net magnetic moment should be undoubtedly attributed to the charge transfer that occurs through Au-S bond. It is worth it to remark that magnetic hysteresis is observed at RT, which implies that magnetic moments should be frozen due to an enormous local anisotropy field; i.e., the anisotropy constant should be, at least, of the order of $10^{7} \mathrm{~J} / \mathrm{m}^{3}$. This value is estimated by comparing thermal energy, $k_{B} T$, and anisotropy energy, $k V$ ( $k$ stands for the anisotropy constant and $V$ for the volume occupied by surface Au atoms bonded to thiol). The estimated $k$ values are summarized in Table I and should be taken as a lower limit since it has been considered a temperature of $T=300 \mathrm{~K}$.

The thermal dependence of the magnetization also points out that Au GNPs behave in a very peculiar way

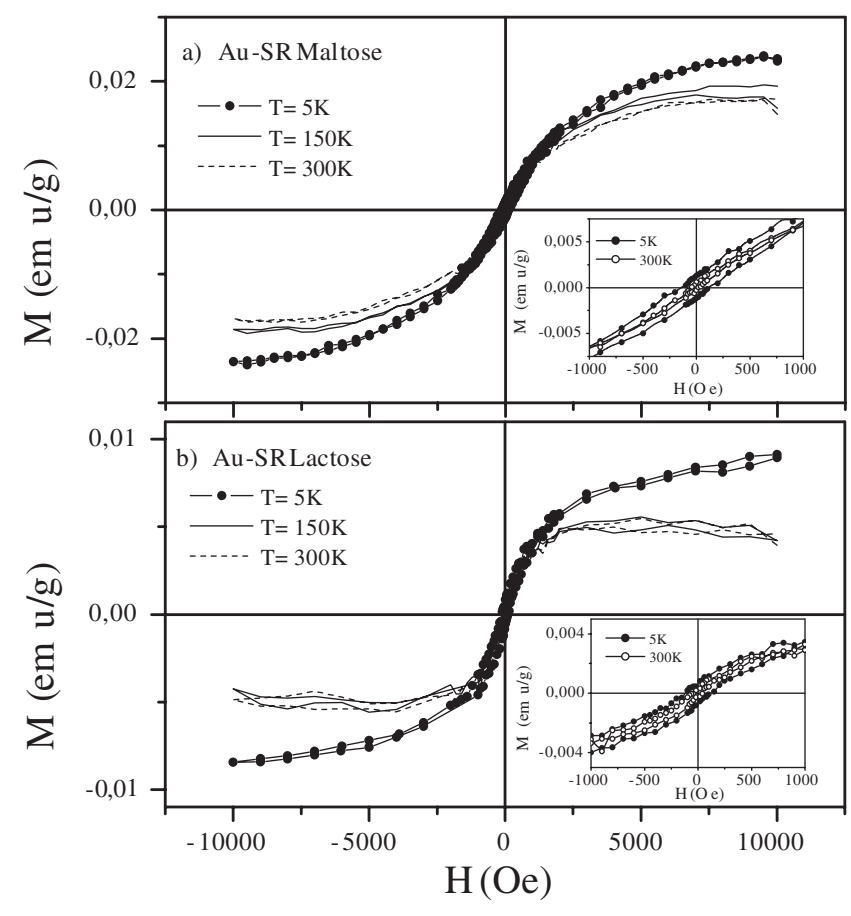

FIG. 1. Magnetization curves of (a) Au-SRMaltose and (b) Au-SRLactose. The inset shows the hysteresis even at $300 \mathrm{~K}$. 
since they exhibit very little dependence with temperature (Fig. 2), remaining almost constant up to $300 \mathrm{~K}$. The lack of thermal demagnetization process points out that exchange interactions between the magnetic moments, if present, are not the main mechanism to be invoked to account for the ferromagnetic-like behavior exhibited by thiol capped Au GNPs, but rather an extremely high local magnetic anisotropy that blocks the magnetic moments.

The magnetic behavior changes considerably upon $\mathrm{Fe}$ impurities addition. Figure 2 compares the magnetization thermal dependence for AuFe GNPs with that of Au GNPs. The magnetization is normalized to the weight percentage of $\mathrm{Fe}$ and estimated $\mathrm{Au}$ atoms bonded to thiol. It is clear that $\mathrm{Fe}$ impurities modify the thermal dependence of magnetization, but not as expected. Although low temperature magnetization increases with increasing Fe content, it is inferred that $\mathrm{Fe}$ impurities are hardly responsible for the anomalous thermal dependence found in Fe free samples. For the three sets of AuFe GNPs, a sharp decrease of the magnetization at very low temperatures is observed that can be attributed to the Fe impurities behaving as para- or superparamagnetic. For very small Fe additions (maltose samples), the magnetization exhibits a further decrease upon increasing temperature, reminding that of a Curie temperature, around $180 \mathrm{~K}$ and $75 \mathrm{~K}$ for AuFe1SRmaltose and AuFe2-SRmaltose, respectively. In the case of AuFe-SRLactose sample, where the amount of Fe is much higher, it is observed that the magnetization values

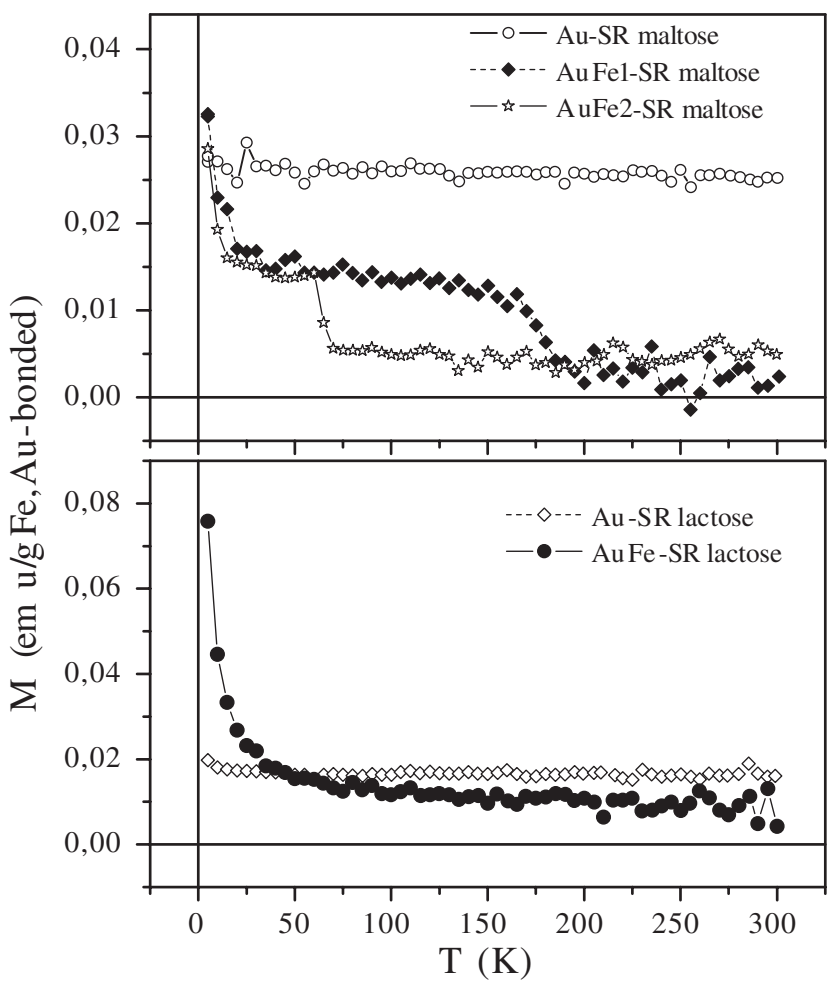

FIG. 2. Thermal dependence of the magnetization for $\mathrm{Au}$ and AuFe GNPs measured under an applied field of 500 Oe. are smaller than for Au-SRLactose for temperatures above $75 \mathrm{~K}$, showing a stronger temperature dependence. Moreover, magnetization curves for the three sets of AuFe NPs also point out that Fe impurities do not enhance hysteresis, but contribute to its disappearance, whereas they enhance the magnetization at $5 \mathrm{~K}$. Figure 3 shows the magnetization curves for AuFe2-SRmaltose and AuFeSRLactose samples. In all cases, hysteresis is observed at $5 \mathrm{~K}$, although the values of coercive field are much smaller than for Au GNPs. The sample with lower Fe content exhibits the highest coercivity at low temperatures. An estimation of the anisotropy constant is summarized in Table I. In contrast with the behavior of Au GNPs, at $300 \mathrm{~K}$ a linear dependence of the magnetization with applied field is observed for AuFe GNPs.

The main conclusion is that $\mathrm{Fe}$ impurities reduce the strong local anisotropy field present at surfaces of Au NPs. However, the mechanism responsible for the weakening of the FM behavior by the presence of $3 d$ impurities is not clear. The differences between the AuFe-SRmaltose and AuFe-SRlactose samples could arise from the different $\mathrm{Au}: \mathrm{Fe}$ ratio. AuFe is a binary system characterized by a high enthalpy of mixing and little mutual solid solubility. For instance, at $200{ }^{\circ} \mathrm{C}, \mathrm{Fe}$ can be dissolved into Au up to a 2 at.\%. According to the Au:Fe ratio, it can be expected that in AuFe-SRmaltose samples, Fe is almost fully dissolved into the $\mathrm{Au}$ NPs forming some kind of $\mathrm{Au}(\mathrm{Fe})$ dilute phase, whereas for AuFe-SRLactose small clusters of Fe atoms that behave as superparamagnetic could coexist together with the diluted $\mathrm{Au}(\mathrm{Fe})$ phase, contributing to the magnetization but not to the hysteresis.

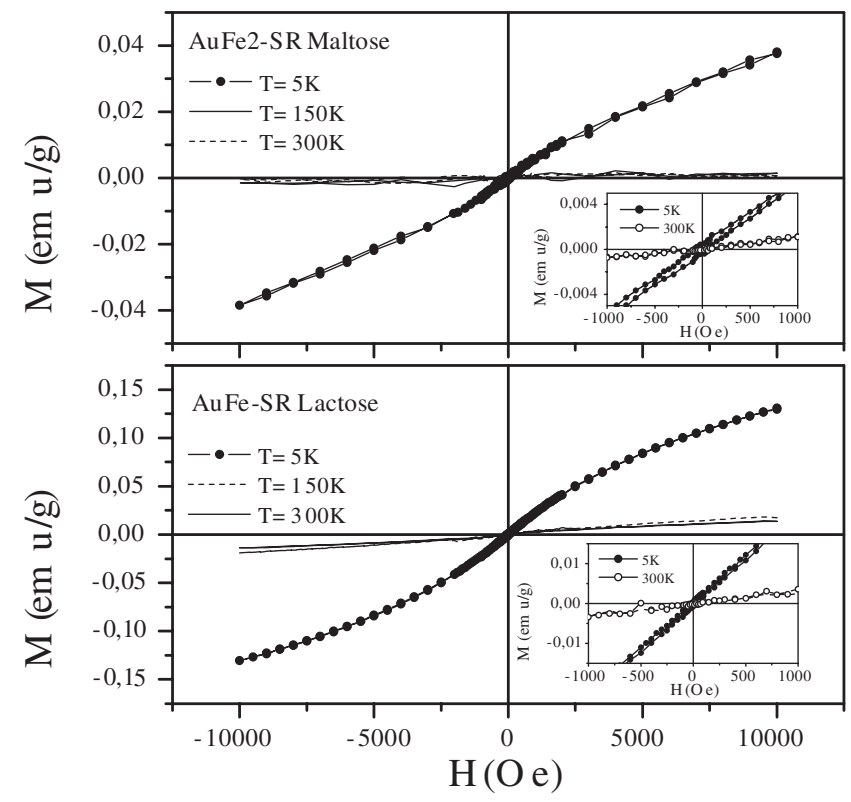

FIG. 3. Magnetization curves of AuFe GNPs. Notice that in contrast with the behavior exhibited by Au GNPs, hysteresis is not observed at $300 \mathrm{~K}$. 


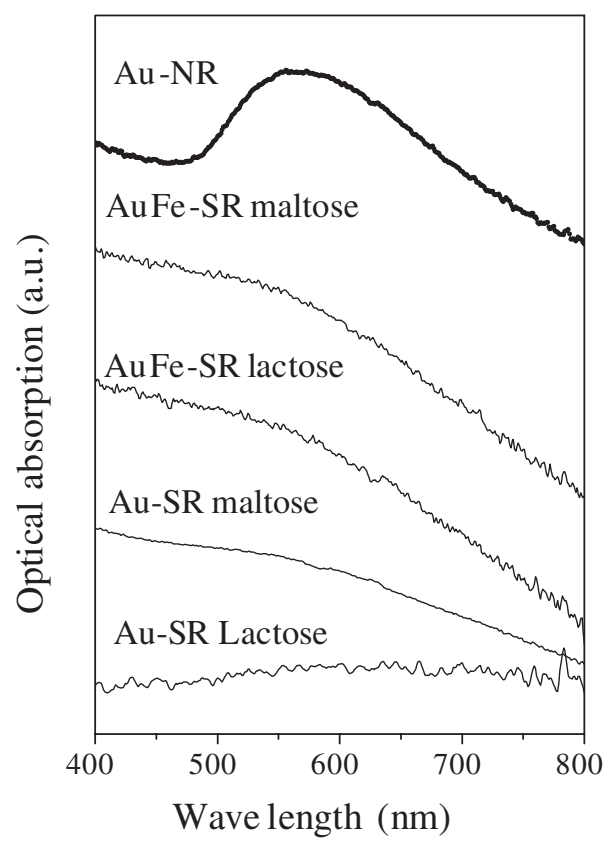

FIG. 4. UV-Visible absorption spectra for the $\mathrm{Au}$ and AuFe GNPs. The spectrum of Au NPs stabilized by surfactant (that exhibit a diamagnetic behavior) is also included.

SPR measurements are shown in Fig. 4. The spectrum corresponding to 2-3 $\mathrm{nm}$ bare Au NPs dispersed with a surfactant (Au-NR) which exhibits a diamagnetic behavior it is also shown. The later shows a broad absorption band centered at about $550 \mathrm{~nm}$ associated to the so-called surface plasmon resonance [14], due to the itinerant character of the $5 d$ holes in diamagnetic Au NPs. This band becomes broader for the AuFe GNPs and is almost suppressed in the case of Au GNPs. These features are an indication of the increase of the damping of electrons due to charge localization at the surface [9] promoted by the strong interaction between surface $\mathrm{Au}$ atoms and thiol capping molecules. The presence of Fe promotes delocalization with respect to Au GNPs. Since the peculiar magnetism observed at nanoscale for Au GNPs has been shown [1] to be associated with localized moments, the effect of $\mathrm{Fe}$ is to reduce this permanent magnetism. However, the paramagnetic magnetization of $\mathrm{Fe}$ atoms contributes to the overall magnetization, mainly at low temperatures, but follows a Curielike law as observed in Fig. 2.

It has been shown that Fe impurities weaken the FM-like behavior exhibited by thiol-capped Au GNPs. Impurities reduce the spontaneous magnetization as well as the strong local anisotropy that blocks the bond-induced magnetic moments. The FM-like behavior in thiol-capped Au NPs has its origin on the charge transfer due to the strong interaction between surface Au-capping molecules, in combination with charge localization and the high value of the spin-orbit coupling in gold surfaces. The almost temperature independent value of the magnetization observed in the Au GNPs samples indicates that the thermal demagnetization process does not follow the typical law that applies in FM elements. In consequence, other mechanisms different from that of the exchange interactions should be invoked. In fact, coupling of orbital momenta have been recently proposed as to be the origin of FM-like behavior in thiol-capped Au NPs [10].

In summary, ferromagnetic behavior observed in thiolcapped Au NPs cannot be ascribed to the presence of magnetic impurities, that, opposite to what is expected, tend to destroy the ferromagnetic behavior.

Support by Spanish MEyC Projects Nos. NAN200409125-C07 and MAT2005-06119 is acknowledged.

[1] P. Crespo et al., Phys. Rev. Lett. 93, 087204 (2004).

[2] I. Carmelli et al., J. Chem. Phys. 118, 10372 (2003).

[3] A.P. Alivisatos, Science 271, 933 (1996); I. Coulthard, S. Degen, Y. J. Zhu, and T. K. Sham, Can. J. Chem. 76, 1707 (1998).

[4] P. Zhang, T. K. Sham, Appl. Phys. Lett. 81, 736 (2002).

[5] H. Liu et al., Phys. Rev. B 72, 155430 (2005).

[6] R. Litran et al., Phys. Rev. B 73, 054404 (2006).

[7] C. Lopez Cartés et al., J. Phys. Chem. B 109, 8761 (2005).

[8] S. LaShell, B. A. McDougall, and E. Jensen, Phys. Rev. Lett. 77, 3419 (1996).

[9] M. A. García et al., Phys. Rev. B 72, 241403(R) (2005).

[10] A. Hernando, P. Crespo, and M. A. García, Phys. Rev. Lett. 96, 057206 (2006); A. Hernando et al., Phys. Rev. B 74, 052403 (2006).

[11] Water soluble AuFe glyconanoparticles with different $\mathrm{Au}: \mathrm{Fe}$ ratio have been prepared as contrast agents for magnetic resonance imaging by reduction in water of a mixture of gold and iron salts in the presence of thiolfunctionalized neoglycoconjugates, J.M. de la Fuente et al., J. Phys. Chem. B 110, 13021 (2006).

[12] A. G. Barrientos et al., Chem. Eur. J. 9, 1909 (2003).

[13] M. J. Hostetler et al., Langmuir 14, 17 (1998).

[14] U. Kreibig and M. Völlmer, Optical Properties of Metal Clusters (Springer-Verlag, Berlin, 1995). 